\title{
Research on small-scale sand fracturing and puff technology
}

\author{
Qian Wang*, Zuohao Wu, Jiapeng Zheng, Junkai Lu, and Menghong Yu \\ Drilling \&Production Technology Research Institute of Jidong Oilfield, Tangshan, China
}

\begin{abstract}
Affected by complex fault blocks, sedimentary environment and reservoir physical properties, Jidong Oilfield generally develops small-scale sand bodies, accounting for $24.2 \%$ of the produced reserves of medium and low permeability reservoirs. Such sand bodies rely on elastic energy to develop and are difficult to use effectively. In this paper, through the three-dimensional hydraulic physical model experiment and the analysis of the oil layer-dry layer-mudstone mixed sand body, the reasons that affect the model parameters are obtained. The results show that: the length of the high-permeability reservoir area, the length of the intermediate-permeability dry layer area, the rate of change of permeability parameters, the viscosity of the construction fluid, and the construction displacement have an impact on the effect of fracturing enhanced injection energy storage.
\end{abstract}

\section{Introduction}

Small-scale sand bodies refer to sand bodies whose scale is small and the existing well pattern is difficult to establish an effective displacement relationship. Affected by complex fault blocks, sedimentary environment and reservoir physical properties, Jidong Oilfield generally develops small-scale sand bodies, accounting for $24.2 \%$ of the produced reserves of medium and low permeability reservoirs. Such sand bodies rely on elastic energy to develop and are difficult to use effectively. It is necessary to strengthen technical research on development methods to achieve beneficial development. According to the characteristics of small sand bodies in G12 block of Jidong Oilfield, a simulation study of crack propagation was carried out. On this basis, a deterministic modeling method was used to establish a fine geological model of the block's work area, and on the basis of cutting and coarsening and reservoir numerical simulation, guided by reservoir numerical simulation results, establish fracturing model, obtain fracturing construction parameters, and guide fracturing design and construction.

\section{Three-dimensional hydraulic model experiment}

This experiment uses a large-scale full three-dimensional hydraulic fracturing physical simulation experimental system. The size of the experimental rock sample is 762 $\mathrm{mm} \times 762 \mathrm{~mm} \times 914 \mathrm{~mm}$, which can effectively reduce the boundary effect of fracture propagation. The system consists of four parts: a rock sample loading frame, a confining pressure loading system, an injection system, and a sound wave monitoring system. The rock sample is placed in the loading frame. The vertical stress is loaded by jack hydraulics, up to $35 \mathrm{MPa}$, and the horizontal ground stress passes through The pressure plate placed in the gap between the rock sample and the frame is loaded. Water is injected into the pressure plate. The increase in water pressure causes the pressure plate to expand. Then the water pressure is transmitted to the surface of the rock sample to achieve the purpose of simulating horizontal ground stress, up to $69 \mathrm{MPa}$.

The injection system is controlled by a hydraulic servo system. The maximum injection displacement in the experiment can reach $200 \mathrm{~cm} 3 / \mathrm{s}$, and the maximum injection pressure at the wellhead is $69 \mathrm{MPa}$. The experimental fracturing fluid adopts two systems of slippery water and cross-linked jelly, and it is convenient to observe the fracture morphology afterwards. A certain amount of phosphor particles are mixed into the fracturing fluid. The fluid injection rate of the shale fracturing experiment was controlled at $1-170 \mathrm{~cm} 3 / \mathrm{s}$. The experimental computer control system can record the wellhead pressure, pumping displacement, and three-way confining pressure data in real time, and can adjust the pumping rate in real time according to the experimental conditions.

The macrophysical model experiment of the rock with natural fractures shows that a number of fractures that are perpendicular to the horizontal bedding and extend along the north-south direction are formed in the open-hole section, and stop at a large natural bedding to the north, and a few cracks extend out of the rock to the south. Like the boundary, part of the fracturing fluid flows along the southern horizontal bedding. Therefore, in the vertical direction, the vertical expansion of hydraulic fractures is restricted by the horizontal bedding, but while communicating with the horizontal bedding, it will also further communicate with the vertical fractures in other parts, which is conducive to the complication of fracture morphology and increases the volume of reservoir reconstruction.

\footnotetext{
* Corresponding author: 1s_wq@petrochina.com.cn
} 
The large-scale model experiment of the rock without natural fractures shows that the fractures form simple fractures with double wings, there are no existing complex fractures, and the reservoir reconstruction volume is limited, see Figure 1.

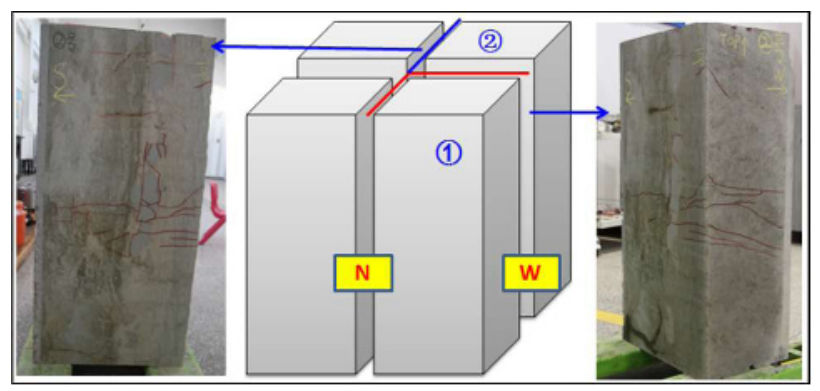

Figure 1. Fracture morphology with natural fractures

At the same time, it can be seen through digital simulation software that: reservoirs without natural fractures form a single fracture; reservoirs with natural fractures can form complex fractures. Numerical simulation shows that natural cracks are the most critical factor in the formation of complex cracks, see Figure 2,Figure 3.

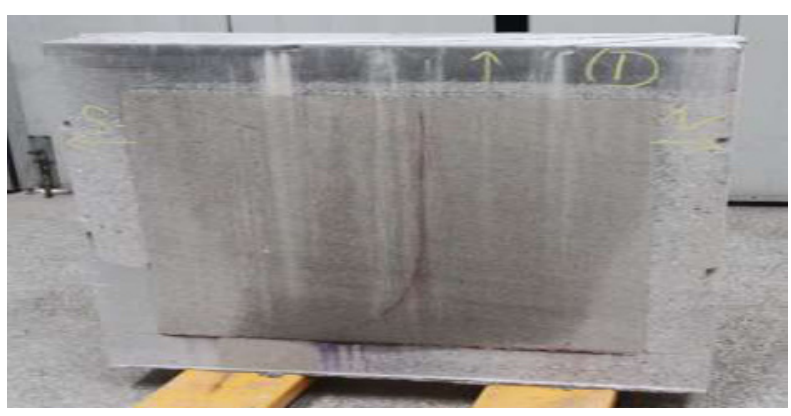

Figure 2. Fracture fracture morphology without natural fractures
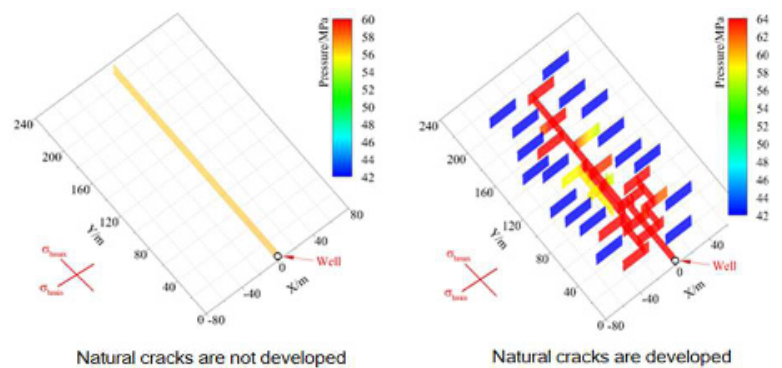

Figure 3. Fracture shape simulated by digital simulation

\section{Oil layer-dry layer-mudstone mixed sand body}

Through triaxial large-scale physical model experiments, it has been pointed out that small-scale sand bodies have the corresponding characteristics, except for the mechanical behavior of artificial fractures when they pass through weak structural surfaces such as natural fractures. When the reservoir physical properties are discontinuously distributed, different process parameters will also lead to different fracturing fluid injection pressurization effects. In order to study the effect of fracturing injection enhancement under different reservoir physical conditions, the fracture extension effects and injection enhancement effects of the reservoir physical properties along the fracture extension direction of oil layer, dry layer, mudstone layer, and oil layer directly contacting the mudstone layer were simulated.

\subsection{Oil layer-dry layer-mudstone transition type}

The difference in reservoir physical parameters is mainly reflected in the difference in permeability, porosity and lithology of the formation. During the simulation, the simulation results of oil layer-dry layer-mudstone layer were compared with the simulation results of oil layer-dry layer-dry layer, so as to analyze the blocking effect of mudstone on fracture propagation. It can be seen from the two figures that when ignoring the existence of mudstone intervals, the extension of fractures is almost unimpeded, the fractures extend longer, and the formation pressure changes caused by injection increase only appear corresponding to the permeability of different intervals. Characteristics. When considering the existence of the mudstone interval, the interface between the dry layer and the mudstone layer has an inhibitory effect on the fracture extension, so that the fracture no longer extends forward, and the injected fluid mainly increases the pressure in the width direction of the fracture in the reservoir. Makes the distribution of the pressurized area more even and a larger area.

From the perspective of fracture geometry, when the existence of mudstone layer is not considered, the fracture continues to extend before, and the length and width of the fracture are both increased. When considering the existence of mudstone layers, the extension of fractures is blocked, and the length and width of the fractures are small. The fluid is mainly used for filtration, thereby increasing the pressure of the reservoir and enhancing the elastic energy of the reservoir,see Figure 4.

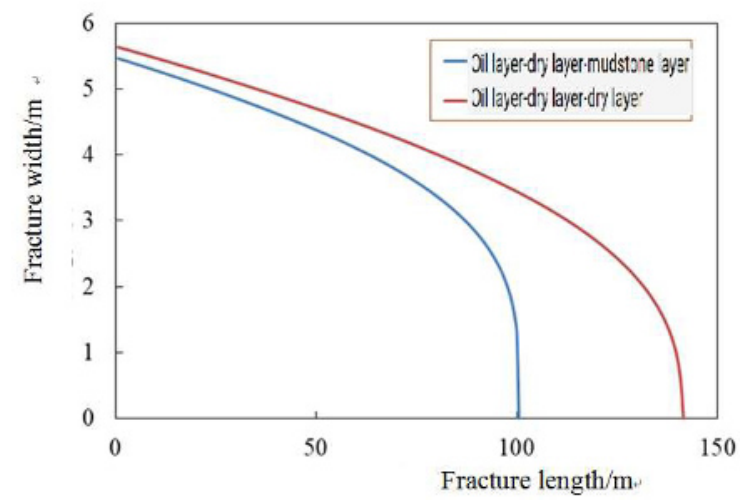

Figure 4. Fracture geometry of oil layer-dry layer-mudstone transition sandbody

The simulation results of the oil layer-dry layer-mudstone transition type show that when there is a variation in permeability in the oil layer, the pressure presents an obvious step-like shape. When the cracks extend and encounter mudstone, due to the poor porosity and 
permeability of the mudstone, it is difficult for the pressure to diffuse effectively in the mudstone, and the pressure diffusion occurs at the sand-shale interface and mainly formed in the dry layer, which is useful for improving the elasticity of the formation. Energy is unfavorable.

\subsection{Oil layer-mudstone mutation}

Further study the effect of oil layer-mudstone breakthrough type fracturing injection. The existence of the interface between oil layer and mudstone will affect the effect of fracture extension. The performance is that when the mudstone exists, the extension of the fracture is hindered, while the extension of the fracture is not considered when the mudstone is not considered. interference. When the fracture extension is blocked, the injected fluid along the sand-shale interface enhances the pressurization effect at the far end of the well, and the elastic energy inside the oil layer increases better; when the fracture extends normally, the injected fluid has a poor pressurization effect on the formation at the far end of the well. And it is mainly used to enlarge the vicinity of the crack tip.

It can be seen from the fracture geometry of the two that the existence of the mudstone layer reflects the influence on the length of the fracture, and the difference in the width of the fracture between the two is proportional to the distance from the wellbore within the fracture. This is because the abrupt change in permeability inside the sandstone caused by the dry layer is not considered at this time, so that the difference between the two is mainly concentrated at the front of the fracture,see Figure 5.

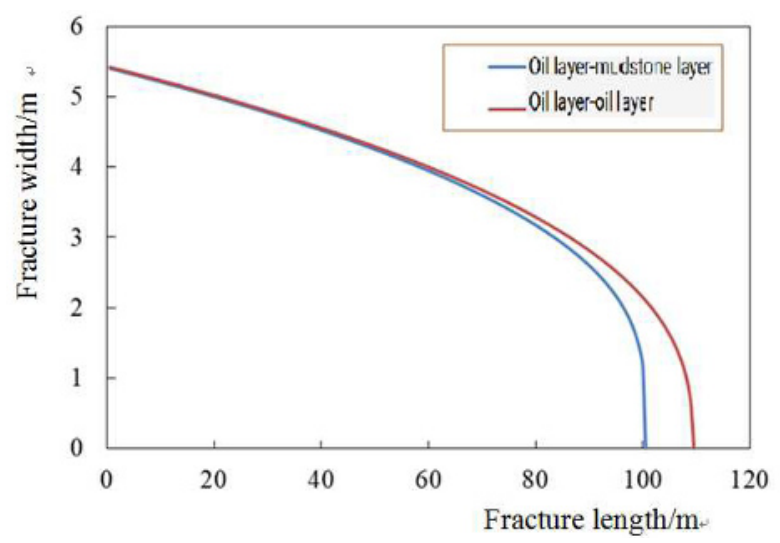

Figure 5. Fracture geometry of oil layer-mudstone catastrophic sandbody

The same study on the oil layer-mudstone abrupt layer shows that after the fracture extends into the mudstone, the pressure spreads along the abrupt interface accelerates. It can be inferred that the conductivity of the far end of the oil well will be improved to a certain extent, which is conducive to water injection and injection. Gas vomiting operation.

\section{Model parameter influence analysis}

In order to optimize the design of the construction of fracturing enhanced energy storage, the influence of different parameters on the effect of fracturing enhanced energy storage was studied. These parameters include the length of the high-permeability oil zone, the length of the intermediate-permeability dry zone, and the permeability Parameter change speed, construction fluid viscosity and construction displacement, etc.

\subsection{Length of high permeability zone}

When the length of the high-permeability oil zone is $50 \mathrm{~m}$, $35 \mathrm{~m}$, and $20 \mathrm{~m}$ respectively, as the length of the highpermeability zone becomes longer, the energy-enhancing effect of the corresponding high-permeability zone becomes more obvious. On the premise of not changing the length of the dry zone with moderate permeability, and when the fracture extends to the mudstone interface, the longer the length of the high permeability zone, the better the supplementary effect of fracturing injection on the elastic energy of the formation, and the increase in the position of the fracture front The better the energy effect.

\subsection{Length of low permeability zone}

When the length of the low- and medium-permeability dry zone is set to $50 \mathrm{~m}, 35 \mathrm{~m}$, and $20 \mathrm{~m}$, the length of the lowpermeability zone has an impact on the injection enhancement effect, but the effect is not as obvious as that of the change in the length of the high-permeability zone.

\subsection{Permeability change rate}

The influence of the change rate of formation permeability in the reservoir on the pressurization effect of fracturing energy storage is studied. The abrupt change in permeability is considered to be that the permeability changes more drastically, while the gradual change means that the permeability changes more slowly. When the permeability changes greatly, the formation pressure distribution during fracturing pressurization has an obvious step-like shape. When the permeability change is small, the change area of the formation pressure distribution cloud map is smooth, and the slower the change speed, the smoother the pressure distribution. At the same time, when the rate of change of permeability becomes slower, it can be seen that the distribution area of formation pressure near the fracture tip is smaller, indicating that the effect of fracturing energy storage and pressurization at this time has become worse.

\subsection{Viscosity}

The fracturing is the influence of fluid viscosity on the construction effect. The higher the fluid viscosity, the greater the pressure raised in the formation, and it is mainly concentrated at the end of the fracture close to the wellbore. When the fluid viscosity is low, the increase in pressure in the formation becomes smaller, but the corresponding distribution area is increased, so that the sweeping range is more uniform, which is more beneficial for recovering the elastic energy of the reservoir. 


\subsection{Displacement}

Under the premise that the total liquid volume of the construction remains unchanged, the influence of the construction displacement on the fracturing energization effect is studied. When using high displacement liquid injection, the maximum pressure increase in the formation is greater, and the pressure The distribution of the lifting distribution area along the cracks is also more uniform, which shows that increasing the construction displacement can increase the pressure diffusion effect at the far end of the cracks, and the effect of supplementing energy becomes better.

\section{Conclusion}

The large-scale model experiment of the rock without natural fractures shows that the fractures form simple fractures with double wings, there are no existing complex fractures, and the reservoir reconstruction volume is limited.

At the same time, it can be seen through digital simulation software that: reservoirs without natural fractures form a single fracture; reservoirs with natural fractures can form complex fractures. Numerical simulation shows that natural cracks are the most critical factor in the formation of complex cracks.

Through triaxial large-scale physical model experiments, it has been pointed out that small-scale sand bodies have the corresponding characteristics, except for the mechanical behavior of artificial fractures when they pass through weak structural surfaces such as natural fractures. When the reservoir physical properties are discontinuously distributed, different process parameters will also lead to different fracturing fluid injection pressurization effects.

In order to optimize the design of the construction of fracturing enhanced energy storage, different parameters have an impact on the effect of fracturing enhanced energy storage. These parameters include the length of the high-permeability reservoir area, the length of the intermediate-permeability dry layer area, and the permeability parameters. Change speed, construction fluid viscosity and construction displacement, etc.

\section{References}

1. Zhang Min.Fine description of the single sand body and the division of reservoir units in the third member of the east 3rd member of the Cha 71 fault block in Chaheji Oilfield [D]. Northwest University 2019

2. Li Yichao.Single sand body description and the corresponding relationship between injection and production in Baima Central District [D]. China University of Petroleum (Beijing) 2018

3. Guo Xulin.Reservoir characteristics of Lianhua oil layer in block Gao 3-6-18 of Liaohe Oilfield[D]. Yangtze University 2019

4. Liu Wei.Single sand body characterization of Baijiantan Formation in the Seven Middle East Area of Junggar Basin[D]. Yangtze University 2019
5. Xiong Zhaochuan.Single sand body fine description and three-dimensional geological modeling research of Chang 61 reservoir in S392 oil field [D]. Northwest University 2018

6. Li Hang.Recognition of thin-bed sand single sand body and analysis of water-flooded characteristics in the outer front of the delta of the Sa II and III oil layer groups in the eastern area of Xingshi District, Daqing Oilfield [D]. Northeast Petroleum University 2018

7. Xiang Yao.Research on the internal architecture model of a meandering channel single sand body based on dense coring wells [D]. Northeast Petroleum University 2018

8. Han Lingling.Research on fine division of sedimentary units and potential tapping of remaining oil in P108 block [D]. Northeast Petroleum University 2018

9. Wang Wen.Reservoir geological characteristics and reserves evaluation of Kumkol South oil field in Kazakhstan [D]. Yangtze University 2012

10. Wang Beibei.Study on the architecture of Chang 6 sandbody in the southern area of Wanggoumen, Wuqi Oilfield [D]. Xi'an Shiyou University 2018 